\title{
Niugini: una nueva nación cuprífera en el Pacífico se acerca a su independencia
}

\begin{abstract}
J A MES B Y TH es Consejero-investigador del Presidente de la Bougainville Copper Pty. Ltd., Melbourne, Australia. Realiza frecuentes visitas a Niugini.
\end{abstract}

En diciembre de 1973, o algo después, veremos surgir una nueva nación en el escenario mundial. Papua Nueva Guinea, un grupo único de territorios y pueblos, alcanzará el místico estatuto de "gobierno autónomo". Se espera que dos años más tarde declare su independencia de Australia, quien es actualmente su tutor colonial y el fideicomisario de las Naciones Unidas.

Tendrá los mismos problemas de todo país en desarrollo, contando, no obstante, con beneficios y refuerzos envidiables al ocupar una posición privilegiada, entre Australia y el Japón, cerca de Indonesia y de los minúsculos estados que surgen en Oceanía. Está además, entre Asia y el Océano Pacífico.

Esta incipiente nación tiene sus propios líderes, elegidos por un sistema tipo Westminster, corregido y con alternativas diferentes, de modo que unifique las tendencias de una opinión pública que no está acostumbrada a las realidades de un país independiente.

Posee su propia bandera pero aún no tiene himno nacional ni un nombre establecido; el de Niugini, favorito de muchos miembros del principal partido político, parece probablemente definitivo. Su población alcanza los 2,5 millones de habitantes, repartida en grupos comunitarios y tribales. En esta nación se hablan 700 lenguajes diferentes, muchas veces incomprensibles entre sí. Por ello existe una permanente disputa por la preponderancia de uno u otro, aunque como medio popular de comunicación se utiliza un inglés chapurreado que, en su desarrollo y al mezclarse con lenguas nativas, ha originado un idioma neo-melanésico llamado Pidgin.

Además, como muchos países en desarrollo, tiene un sentimiento separatista latente que se une a la tradicional burocracia colonial centralizada. En sus aspiraciones y determinaciones la nueva nación pareciera alejarse de las orientaciones del anterior gobierno colonial, pero las realidades económicas aseguran la continuidad de los estrechos vínculos comerciales con aquellos poderes a quienes ha estado 
ligado históricamente, tanto en la guerra como en la paz: Australia y el Japón.

Como observadores amistosos, buscaremos clar una visión de Niugini, como una nación, que teniendo ya diseñadas sus prioridades nacionales quiere erigir sus nuevas estructuras en los mejores fundamentos del gobierno colonial que tuvo anteriormente, dejando de lado sus aspectos destructivos.

Frente a este panoiama confiado, hay' gente que duda. No se trata de personas que se dejen guiar por las coloridas descripciones de periodistas y estrategos de salón, sino de académicos que, como indicó uno de ellos vivamente interesado por el proyecto: "Posiblemente este sea uno de los accidentes que se repiten en la historia, de corta vida, basado en las ilusiones de un poder pequeño (Australia) que se expande dentro del área, en un periodo en que las realidades del poder se encontraban disfrazadas por el sistema colonial. La nueva balanza del poder recién toma hoy su auténtica forma; seguramente rediseñando nuevas fronteras". ${ }^{1}$

De acuerdo a este autor, podemos esperar que tal sea la peor posibilidad. Pero también es posible que un modelo de desarrollo elegido libremente logre levantar a una nación vecina de Australia y Nueva Zelandia, con sus propias pautas y preferencias, con mayores riquezas, en el comienzo de su vida independiente, que muchas otras naciones en desarrollo del mundo.

Siendo Papua Nueva Guinea un país del cinturón de fuego del Pacífico, es geológicamente reciente $\mathrm{y}$ tiene volcanes en actividad y muchas áreas propensas a los terremotos y temblores. Los xíos son erráticos y cambiantes. Su territorio es escaxpado, abrupto e inestable para la construcción de caminos.

El medio ambiente de la población tiene gran variación, yendo desde las islas y playas con pueblitos de pescadores que recuerdan aquellos polinésicos, hasta los poblados de los pantanos donde la palma de "sago" es la principal fuente de alimentación, o también aquellos remotos caseríos de las serranías donde la papa (kau-kau en dialecto Pidgin) constituye la dieta habitual de sus moradores.

Ciertamente, este es un país de horticultores y recolectores. Las batatas, el taro, yams y el sago son los productos más abundantes. Las bananas y los cocos son los principales alimentos en ciertas regiones, teniendo gran valor los frutos de los árboles. "El cerdo es la única fuente animal de proteínas, personaje principal de las fiestas, de los regalos. Se lo suele ver vagando en busca de alimento o criado por las mujeres hasta su ceremonioso final. Son fuente de continuas

${ }^{2} \mathrm{C}$. D. Rowley, El aldeano de Nueva Guinea: observaciones desde 1964. Cheshire, Melbourne, edición 1972, p. 9. 
disputas, ya sea por las depredaciones que causan como por su posesión."

Los dirigentes políticos de paises recientes, como Niugini, están en distinto plano a los de los países sudamericanos, quienes cuentan con una larga etapa de colonización y poseen una formación jurídica definida. "Representan a pueblos que previamente al establecimiento de la administración colonial vivían en comunidades de pueblos o tribus, o en grupos pequeños ligados por el parentesco; no existía el estado, ni instituciones para ejercer la justicia ni leyes redactadas, sin más protección que la del parentesco, ni lenguaje escrito, administración impersonal, ni economía monetaria o medios de ahorrar para mejorar el nivel de vida. Poseían una determinada forma de ser y sus fronteras, que les fueron delimitadas por un poder colonial que los agrupaba dentro de unidades para administración. Los colonizadores utilizaron a estos dirigentes para sus propios fines, pero también en cierto sentido los formaron, dándoles unidad".3

\section{LA HISTORIA HASTA 1962}

Nueva Guinea figuró por primera vez en la historia de Euxopa como resultado de la exploxación hispánica. Es muy probable que Jorge de Meneses, el capitán delegado a las Molucas, haya recalado en la isla principal en 1526. El interés de España por las islas de las Especierías, sin embargo, resultó en definitiva más importante. Alvaro de Saavedra navegó la costa norte antes de su muerte, en 1529. En 1545 Iñigo de Ortiz de Retes llamó por primera vez Nueva Guinea a la región. En 1567 Alvaro de Mendana navegó hacia el oeste, partiendo de Lima, para buscar las "Islas de Salomón". La expedición descubrió y denominó a las islas que se encuentran al sud-este de la actual Nueva Guinea, y en 1595 Mendana estableció un poblado que fracasó, en la isla de Santa Cruz.

$\mathrm{Su}$ piloto mayor, Pedro Fernando de Quirós, "estaba obsesionado con la idea de descubrix el continente austral y salvar las almas de sus habitantes de la perdición." ‘ Volvió a las islas en 1606. Su segundo de a bordo, Luis Váez de Torres, navegó a lo largo de la costa sur de Nueva Guinea Oriental, que hoy lleva su nombre.

Exploraciones más avanzadas de navegantes holandeses, franceses

${ }^{2}$ C. D. Rorwley, op. cit., p. 26.

${ }^{3}$ C. D. Rowley, op. cit., p. 4.

${ }^{4}$ C. Jack-Hinton, "Descubrimiento". Enciclopedia de Papua Nueva Guinea, Melbourne University Press, 3 vol., 1972, p. 251 b. 
y británicos se siguieron realizanclo en los 200 años posteriores. La Isla de Bougainville fue descubierta por Louis Antoine de Bougainville en 1768. Treinta años pasaron hasta que se conocieron los rasgos principales del archipiélago, a lo cual seguiría una colonización europea efectiva.

En 1828 los holandeses reclamaron la porción occidental de Nueva Guinea, limitándola al este por el meridiano 141 de latitud Este. Los holandeses lo administraron hasta 1962 y en 1963 se convirtió en Irian Occidental, que es una parte de Indonesia.

El resto de Ia isla, hoy llamada Papua Nueva Guinea, estuvo bajo el mandato británico y germánico en el apogeo clel imperialismo colonial occidental. La primera instalación permanente de Europa en la isla principal fue en 1874, cuando el Reverendo W. G. Lawes, de la Sociedad Misionera de Londres, se estableció en lo que hoy es Port Moresby, la capital de la nación, situada al sur del territorio converticlo en el presente en Papua. Alemania comenzó a interesarse por la región en 1884, cuando se fue abriendo campo dentro del mundo de los grandes poderes coloniales.

El desarrollo germánico se centró en el territorio del norte e islas cercanas. Lo dirigió una compañía de fletes, hasta 1899; resultó una historia de insalubridad, ineficacia y pérdida financieras que lo convirtió en una aventura con escasas ganancias. No exa una segunda Java, que pudiese competir con el bienestar colonial de los holandeses. El control administrativo directo de Alemania se centró en Rabaul, la capital colonial situada en la Península de Gazelle, en Nueva Britania. Ista gestión fue metódica y dura, pero le dio un futuro alentador, en 1914.

El interés germánico hizo surgir intensas críticas en las colonias australianas, porque veían a las islas del Pacífico como extensión lógica de la posesión británica. El Primer Ministro de Queensland, la colonia australiana más cercana, envió a un magistrado policial a través del Estrecho de Torres para anexar Nueva Guinea en 1883. El gobierno británico rechazó la ratificación de la anexión. La griteria que se levantó fue causa de que al año siguiente se firmara un acuerdo anglo-germánico para la división de Nueva Guinea entre ellos. El Protectorado Británico comenzó en 1884.

La Nueva Guinea Británica fue, entre 1888 y 1902, una colonia formal. Sólo en 1906 el nuevo gobierno clel Commonwealth Australiano, que había asumido algunos de los poderes estatales particulares, tomó bajo su responsabilidad directa esta área.

Cuando en 1914 estalló la guerra, un contingente naval australiano navegó rápidamente desde Sy'lney para ocupar la Nueva Guinea Germánica y apoderarse de las estaciones de radio. Rabaul fue 
capturado rápiclamente; a continuación vino una administración militar muy especial, duxante los años de guerra.

La armada tenía órclenes de no anexar el territorio germano, por lo cual los dueños de plantaciones alemanas y los misioneros continuaron sin disturbios.

Después de Versailles, Nueva Guinea quedó clasificada como mandato clase C, dentro de la Liga de las Naciones. Papua permaneció separada. Las tres regiones desaryollaron significativas diferencias, tanto en estilo administrativo como en sus leyes, cuyos efectos aún no se han eliminado.

Ambos territorios eran remansos coloniales de vida tranquila donde habian pueblos y áreas de increible lejanía, firmemente administrados a bajo costo por Ios hombres blancos de Australia; la copra (almendra de coco) y otros frutos de recolección, junto con cierto mineral aurifero exan las principales exportaciones. La Liga de las Naciones mantenía una cierta vigilancia sobre Nueva Guinea, no así sobre Papua. Las islas eran una frontera tropical donde incluso aquellas xiquezas que podrian descubrirse serían de difícil desarrollo y explotación.

"Entre la carencia de recursos financieros y la falta de personal adecuado no se había llevado a cabo ninguno de los fines proclamados por la administración australiana, cuando la Segunda Guerra Mundial provocó la eliminación del régimen civil. Las medidas de desarrollo habian producido una economía basada en una sola siembra, la copra, sujeta a las fluctuaciones del mercado mundial. El proteccionismo mantuvo una sociedad preservada dentro de sus pueblos $y$ aldeas tradicionales, pero que sufría de las frustraciones reveladas en un importante movimiento, llamado "Cargo", un culto que afecta a los grupos nativos en un estadio determinado del contacto europeo."

"El Cargo", un culto milenario y a menudo mesiánico, es originario de Niugini y Melanesia. Por medios secretos y siguiendo cierto ritual puede producir Kago, es decir las mercancías importadas duraderas de la civilización occidental. En muchos aspectos es una solución racional elaborada por una sociedad primitiva para explicarse los misterios del cambio occidental y el incumplimiento de las expectativas que surgen. Ellos esperan que, utilizando un ritual apropiado lograrían bienes europeos en grandes cantidades, traídos por avión o barco."

"El logro principal del gobierno de Papua y Nueva Guinea fue el de todo poder colonial que se inicia: el establecimiento de la ley y el orden sobre muchos territorios por medio de la penetración pacifica, acción fundamental en un régimen colonial. Las etapas finales de éste, es decir la diversificación de la economía y el ele- 
vamiento general del nivel de vida primitivo, habian sido sólo ligeramente abordados: y a consecuencia de esta falla relativa en los últimos tramos del desarrollo y bienestar, los dos gobiernos han sido censurados en los años posteriores."

"Dejando de lado los factores externos de guerra y depresiones que obstaculizaban el progreso de los territorios y sobre los que ni el gobierno australiano ni los gobiernos locales tenían mucho control, ese criticismo para censurar una situación como la de Australia con respecto al poco desarrollo de sus colonias, no es justo. Tenemos que consiclerar que una nación en desarrollo, necesitada de capital y de su propia mano de obra no podía movilizar sus recursos hacia las colonias porque ello iría en detrimento propio. Se culpa también a quienes se vieron involucrados en la política colonial con juicios totalmente anacrónicos: se pretende enjuiciar a los hombres y la época anterior a la Segunda Guerra Mundial, con la óptica del mundo de la post-guerra."

En Niugini fue desastroso el impacto de la Segunda Guerra Mundial. Muchos de los cambios siguientes, incluso la misma independencia, pueden delinearse desde los acontecimientos de ese período.

"Entre diciembre de 1941 cuando los japoneses dieron el golpe y agosto de 1945 con su rendición, ocurrieron cambios que sobrepasaron ampliamente todo efecto en la población de la venida primitiva de los hombres blancos, que había sido local y gradual, o de cualquier catástrofe natural, ya sean enfermedades, terremotos o erupciones volcánicas. Aun la guerra misma fue para los nativos un clesastre sin importancia, acostumbrados a ser diezmados por el hambre, las privaciones, el cautiverio y la muerte violenta. Sin embargo, casi todos los cambios experimentados en esas sociedades tribales, hoy llamados "progreso", se originaron en cierto modo a consecuencia de la Segunda Guerra Mrundial."6

El país fue el límite sur de la embestida japonesa en el Pacífico. Se estima que alrededor de 300.000 japoneses servian en el área y que menos de la mitacl de ellos sobrevivieron. Significativamente, 110.000 de ellos fallecieron de hambre e inanición. El ejército australiano perdió cerca de 14.500 hombres, tanto en batalla como muertos por heridas o perdidos. Las tropas americanas también sufrieron muchas bajas. Pero la muerte y el sufrimiento de la población nativa "nunca ha sido descrito en forma completa y ya no conoceremos sus terribles detalles". ${ }^{\top}$

"Francis West, "Los antecedentes históricos", Nueva Guinea en el umbral. Ed. E. K. Fisk, ANU Press, Canberra, 1966, p. 18-19.

"Peter Ryan, "La Segunda Guerra Mundial", Enciclopedia de Papua Nueva Guinea, p. 1.211, f.f.

'Ryan, op. cit., p. 1.223. 
"Se desconoce la cantidad exacta de habitantes asesinados en excursiones aéreas, barcos hundidos o accidentes, o por enfermedades, ya sea en sus pueblos como en el servicio militar junto a los Aliados o a los japoneses. Se ha calculado que, en el período culminante, 55.000 varones mayores de 14 años estaban sirviendo como conscriptos para los americanos y australianos, a menudo en espantosas condiciones."

"La gran variedad y división territorial de Niugini se tradujo, durante la guerra, en un distinto grado de impacto según cada región. Las grandes poblaciones de las tierras altas centrales, todavía lejos del contacto europeo, sólo fueron testigos de algunos vuelos de aviones. Las islas y la costa norte permanecieron varios años bajo la ocupación japonesa, que tuvo una ferocidad variable, y las regiones del interior fueron escenario de espantosos combates. El área sur, Papua, se salvó de la ocupación japonesa aunque también aquí los pueblos quedaron desprovistos de sus varones, quienes sirvieron como obreros o estibadores. La guerra fue, sin lugar a dudas, un acontecimiento que marcó a muchos ciudadanos importantes de hoy'. La población vio horizontes nuevos e inconmensurables. Hubo un cambio radical en la relación primitiva entre el "amo" blanco y el siervo negro: vieron tipos distintos de hombres blancos, los australianos, cuyo sentido cle humanidad, informalidad y buena voluntad para trabajar al sol, dentro del barro, eran un pasmoso contraste con la rigidez y aislamiento de la gran mayoría de los residentes blancos anteriores. Los militares negros de EE.UU. que realizaban trabajos de inteligencias superiores fueron el desmentido a un axioma hasta entonces aceptado, de la inferior capacidad de la raza negra". 8

"Muchos vecinos de Papua Nueva Guinea conocieron por primera vez otras regiones de su país y una gran cantidad visitó Australia. Inclusive lugares remotos como América pasaron a ser una realidad conocida y no sólo un simple nombre."

"La enorme prosperidad material de los americanos y los australianos, demostrada por sus miles de vehículos motorizados, aviones, ropas, alimentos y armas era materia de admiración por parte de la población local, y también fuente cle envidia. Esta fue canalizada en gran parte dentro de corrientes racionales y constructivas, tal la creciente pasión por la educación y una nueva vida política que se proponia lograr la independencia nacional. Algunas corrientes sirvieron para eliminar la estupidez del "Cargo" y el racismo estéril contra el blanco."

"Papua y Nueva Guinea. concitaron el interés y entendimiento de

'Ryan, op. cit., p. 1.223. 
Australia, al terminar Ia guerra. Decenas de miles de australianos en servicio estuvieron allí. Muchos deben sus vidas a la ayuda que recibieron de la población nativa, conociendo su penosa labor al servicio de los Aliados. Teniendo estos antecedentes los gobiernos australianos encontraron políticamente útil hacer desembolsos públicos en Papua Nueva Guinea, en un nivel nunca pensado antes de la guerra y financiar así, en parte, el progreso y desartollo que el pueblo requería."

"Hoy en día, se quisiera volver al tiempo de la economía de recolección, para olvidar los horrores de la guerra. Pero los días soñados de un tranquilo pasado colonial no volverán jamás. Para bien o para mal, la Segunda Guerra Mundial, más convulsiva y penetrante que ningún terxemoto, lanzó a Papua Nueva Guinea dentro de la corriente de rapidez y eficacia del mundo moderno."'

LA ÚLTIMA DÉCADA

Entre el fin de la guerra y 1962 , la política australiana respecto a Papua y Nueva Guinea tuvo un desarrollo gradual. Tomó un peso creciente, financiación y dimensiones durante el largo reinado de Sir Paul Hasluck, Ministro de los Territorios entre 1951 y 1969, y hoy en día Gobernador General de Australia. El plan de desarro11 o graclual de Hasluck suscitó entonces y también hoy, fuertes críticas. Era una política exclusivista; había renuencia a fomentar la asistencia externa y existió un estricto control de visitas permitidas. En el año 1962 comenzó una década de cambios.

En ese año, una misión que visitó los territorios, designada por la N.U. produjo un impacto enorme. Ella llevó al establecimiento de la actual Casa de Asamblea (o mejor dicho Parlamento), a la instalación de una Universidad y más tarde un Instituto Tecnológico. En una época en que las criticas contra el colonialismo iban en aumento en las Naciones Unidas, la misión, dirigida por Sir Elugh Foot del Reino Unido, requirió una revisión completa de la economía por parte del Banco Internacional para la Reconstrucción y Desarrollo. El informe del I.B.R.D. que se obtuvo luego de las investigaciones en terreno, en 1963, contribuyó en gran parte al tipo de desarrollo que se dio en Niugini en la década siguiente ${ }^{10}$.

${ }^{9}$ Ryan, op. cit., p. I.224.

${ }^{10} \mathrm{El}$ informe fue publicado y constituye un importante estudio de los acontecimientos de ese período. El desarrollo económico del Territorio de Papua y Nueva Guinea, IBRD, The John Hopkins Press, Baltimore, 1965. 
Este informe describe a la política practicada por Australia como "paternalismo benevolente" 11 , y también entrega la primera revisión externa del desarrollo del país; algunas de sus recomendaciones fueron aceptadas. El informe Ilevó a un programa de desarrollo quinquenal redactado en 1968 y modificado en 1971. Frente al gobierno de esta nueva nación tenemos la Coalición Nacional, formada después de las elecciones de 1972. Ella está constituida por el Pangu Pati (que favorece la unidad de Papua y Nueva Guinea), como fuerza mayor; por el Partido de Progreso Popular y un grupo de independientes y fuerzas locales. Las divisiones por partidos son menos importantes que sus personalidades.

El desarrollo de un gobierno parlamentario elegido por votación ha ido progresando desde los primitivos asesores del Consejo Legislativo, en un comienzo designados y más tarde elegidos, con restricciones, hasta la actual Asamblea cuyos miembros son elegidos por sufragio universal. Solamente ocho entre cien parlamentarios, en 1972, son europeos con muchos años de residencia en el país; de ellos, solamente uno recibió un ministerio en la distribución inicial de cargos.

La estructura y formalidades de la Casa de Asamblea son una copia de las de Westminster, pero no está claro si de hecho permanecerán de tal modo, en tanto el país desarrolla sus propios modelos y filosofía nacional. La Coalición creó el puesto inexistente hasta entonces, de Jefe de la Oposición; el beneficiado con el cargo expresó que prefiere ser considerado como cabeza de una alternativa de gobierno, ya que el concepto de "oposición leal" tiene una apreciación distinta entre los miembros. Un grupo de los que apoyaron en el Parlamento de 1968 al United Party (hoy en la oposición) se cambiaron de partido en 1972, argumentando que sus electores los habían enviado para estar en el "gobierno". La mayor parte de los discursos contienen pedidos de fondos para caminos, puentes y escuélas. Un Comité de Planificación Constitucional, encabezado ¿ex oficio? por el Primer Ministro, Mr. Michael Somare y en efecto por el joven sacerdote católico, Padre John Momis (nacido en Bougainville), estudia los posibles cambios en la estructura gubernamental y la creación de una constitución; su trabajo puede ayudar mucho al establecimiento de una estructura más viable que tome en cuenta las tendencias separatistas notadas en diversas partes del país, en donde las demandas para un desarrollo local sólo son manifestaciones epidérmicas.

Australia, responsable ante las Naciones Unidas, en diez años ha

${ }^{11}$ F. A. Mediansky, "Relaciones Exteriores", Enciplopedia de Papua Nueva Guinea, p. 395 B. 
pasado descle una posición oficial que retardaba el eventual desarrollo de Nueva Guinea hacia su inclependencia, tema para ella aún lejano, a respaldar el gobierno propio, si se le requiriese. El nuevo ministro del Partido Laborista Australiano (ALP), Mr. Whitlam, molestó a los australianos conservadores por su crítica abierta a las mediclas del Gobierno, en visitas efectuadas cuando estaba en la oposición. Sus criticas y las medidas que anunció en ese entonces han siclo aplicadas por el joven Primer Ministro para los Territoxios Exteriores, Mr. Andrew Peacock, en los últimos años del gobierno liberal, con visos de ser continuados por el nuevo ministro del ALP, Mr. W. L. Morrison quien es un economista, miembro del servicio cliplomático australiano durante 20 años.

Desde hace años la frontera entre Australia y Niugini, el Estrecho de Torres, ha sido fuente de conflictos.

El Commonwealth Australiano tomó en 1901 las fronteras de las colonias previas; la de Queensland, a pesar de los intentos en contra, se extiende a través del estrecho, en dirección a las islas, a distancia navegable de Niugini. Tanto el gobierno de Whitlam en Canberra como la Coalición Nacional en Port Moresby han señalado su preferencia por un movimiento de la frontera hacia el sur; esta moción lia sido rechazada por el Primer Ministro conservador de Queensland, quien ha puesto de relieve el problema de la población isleña, los que, aunque estrechamente ligados al pueblo vecino del territorio de Niugini, son ciudadanos australianos que pagan sus impuestos y reciben los beneficios sociales correspondientes. Los derechos de pesca y los posibles pagos por la propiedad que se deriven de la futura exploración de petróleo submarino forman parte integral del problema, aún sin resolución.

La Coalición Nacional, bajo el mando de Mr. Somare, fijó como fecha probable para el gobierno autónomo el mes de diciembre de 1973. El gobierno australiano dirigido por el ALP quiere acelerar la transición hacia la indepenclencia y está activando la entrega de poderes internos al control de Niugini. La Coalición Nacional, enfrentada a los conservadores rurales teme que la independencia signifique el término de la ayuda australiana y por consecuencia, del desarrollo local. Por tanto, no acepta un plazo fijado unilateralmente por Australia y quiere elegirlo por sí misma. Mientras que la Defensa y los asuntos exteriores siguen teniendo cierta orientación protectora por parte de Australia, a pesar de la independencia, la Coalición Nacional ha establecido claramente su necesaria independencia para tomar varias responsabilidades.

El Ave del Paraíso, uno de los pájaros magníficamente emplumados del mundo, se ha constituido en el símbolo nacional de la nueva nación. Entre 1880 y 1920 llegaban cerca de 8.000 pieles de ellos 
al mercado de plumas europeo. La exportación de aquellas plumas hoy está prohibida por la ley y existen severos castigos por la matanza de aves con armas de fuego, aunque se permiten los antiguos métodos de caza.

En dialecto Pidgin se denomina Kumul a esta ave, que se aplicó en 1972 a la aerolínea nacional proyectada, cuando en un concurso público ganó el nombre "Kumul Air Services"; también fue aceptado este nombre para la futura unidad nacional de moneda que reemplazará al dólar australiano.

Al tiempo de escribix este artículo no se había publicado el valor nominal de la nueva unidad ni se sabía la fecha de introducción. Se suscitó una impresionante discusión sobre los beneficios y dificultades en la introducción de la nueva moneda, entregando el Dr. R. G. May (quien trabaja en el Banco de Reserva Australiano y hoy es director de la Universidad Nacional de Australia en la unidad de Investigación sobre Nueva Guinea) ciertas preguntas sobre su relación actual o futura con el dólar australiano, en septiembre de 197212. El alza creciente del dólar que siguió no mejoró expectativas importantes para el progreso del país, en cuanto al comercio de la copra, del café y de los demás productos tropicales.

Para 1973 se espera que la Coalición Nacional introduzca una legislación sobre las formas de ciucladanía. En la actualidad el status legal de la población se divide en: Papuanos, es decir aquellos nacidos en la mitad sur de la isla principal que tienen categoría de ciudadanos australianos; y los habitantes de Nueva Guinea que son "personas bajo la protección australiana". En la práctica existe muy poca diferencia.

También se ha planteado la doble nacionaliclad para aquellos residentes australianos que llevan largo tiempo en el pais y para quienes nacieron y han vivido allí la mayor parte de sus vidas, pero no se cree que esto sea factible.

El proceso de localización ha sido muy rápido, desde la elección de la Coalición Nacional, en 1972. Se ha formado rápidamente un equipo de gente habilosa y bien educada que se prepara para el servicio público, buscándose por otra parte un retorno de los expatriados al hogar. El Primer Ministro dijo en 1972 que esperaba reducir el número de expatriados (de abrumadora mayoría australiana) del servicio público, aunque todos los proyectos requieren una continua necesidad de miles de extranjeros, especialmente profesores secundarios y técnicos para enfrentar el desarrollo de la próxima décacla. Si bien se ha sugerido llamar personal preparado, a

12Ron May, "Dinexos del cielo - Problemas de una moneda corriente separada", N'ueva Guinea, vol. 7, ni 3, septiembre-octubre de 1972. 
un costo inferior, de otros países en desarrollo que tienen amplia experiencia en materia de avances educacionales, no se han tomado medidas al respecto. También está en debate la educación nacional, ya que tanto la gubernamental como la misional requieren cambios básicos en su curriculum para que se oriente a las necesidades de un país de economía agxícola, diferenciándose del esquema occidental.

En Australia se están formando los primeros diplomáticos de Niugini, habiéndose designado los clos primeros en embajadas australianas. La Coalición Nacional anunció que pretende combinar los asuntos diplomáticos y de negocios en un solo ministerio. Tanto sus intereses como sus modestas dimensiones nacionales se ven claramente demostrados en los primero destinos: Tokyo, Jakarta y Bruselas. Se han realizado visitas ministeriales a las islas vecinas del Pacifico Sur, ya que Fiji, Tonga y la minúscula Nauru lograron su independencia $y^{\gamma}$ lo mismo pueden hacer otros territorios coloniales en la próxima década.

Indudablemente Niugini, a su debido tiempo, deberá tener representación diplomática en Canberra y ante las Naciones Unidas. No existe representación diplomática o consular en Port Moresby: pero se esperan tendencias de acercamiento por parte de Japón, Indonesia, Ios Estados Unidos y posiblemente Gran Bretaña y Alemania Occidental, dentro de tres años.

Al resultar electos, los ministros de la Coalición Nacional se enfrentaron con un país, pero no sólo con sus problemas prácticos y con la necesidad de asumir cada rez más poderes entregados por los australianos, sino con algo muchísimo más importante que es elegix un plan de desarrollo, ya que casi ningún ministro tenía expexiencia previa en asuntos de gobierno.

En junio de 1972, el Primer Ministro, Mr. Michael Somare, presentó un informe básico al Parlamento titulado "Programa para el desarrollo. Principios, elecciones y prioridades". Postulaba la elección alternativa de una economía de crecimiento convencional, o una basada en pequeños porcentajes de crecimiento pero con gran desarrollo del pueblo y participación económica indígena. Luego de un intenso debate intermo dentro de la coalición, el Primer Mrinistro anunció en diciembre de 1972 que presentaría un programa de progreso a la Asamblea en la primera mitad del año 1973. Sus puntos importantes abarcaban la descentralización, el adelanto rural, la distribución equitativa del ingreso y la confianza en el país. La Coalición pareciera favorecer, al menos por principio, una política que le dé énfasis al bienestar social más que al crecimiento económico convencional de Occidente.

El Primer Ministro afirmó que uno de los propósitos planteados seria el control gubernamental en aquellas áreas de la economía 
donde éste es necesario para asegurar el tipo de desarrollo deseado. Lo calificó de este modo:

"Vemos a la inversión extranjera y la cantidad de hombres de negocios privados que se ha extendido muchísimo, como la columna vertebral del sector monetario de nuestra economía. El control estatal y su ingerencia se limitaría a las actividades principales de la nación tales como la aviación, la centralización de la banca o la promoción de inversiones para ciertas partes del pais donde los hombres de negocios de Papua o extranjeros, quienes operan sobre la base de ganancias directas, no tienen interés en participar. Estos propósitos son una clara indicación del cambio de orientación que mi gobierno quiere intentar, para dar prioridad al bienestar social antes que a los aspectos estrictamente económicos del progreso."13

Los países en desarrollo se parecen por varios aspectos; sin embargo, en el caso de Niugini la diferencia visible ha sido que Australia, como poder gobernante, no ha logrado ningún beneficio financiero de su control.

Como lo señalara E. K. Fisk y Maree Tait ${ }^{14}$, seguramente la contribución sin compensación de la ayuda directa por parte de Australia a Niugini excederá en 1980 los 3.000 millones de dólares australianos, lo que significa 1.000 dólares por familia australiana. Desde 1895 la exportación total de mercaderías desde Niugini ha subido ligeramente su valor por sobre 9.000 millones (incluyendo $\$ 100$ millones de dólares australianos re-exportados).

El total retenido por importaciones de bienes durante el mismo período era cerca de $\$$ A 1.200 millones. Ellos esperan que el déficit total en cuenta corriente será cercano a los 1.000 millones de dólares australianos en 1970.

Ambos autores señalan también que esta entrada de ayuda, un regalo anual cercano a los 140 millones de cólares australianos en mercaderías y servicios "son un auténtico y valioso regalo... son mercaderias y servicios que harian bien al propio pueblo australiano". Esto quiere decir que la ayuda australiana no tiene nada que ver con la ayuda norteamericana del Punto Guarto, originada en la superproducción, sino que se trata de bienes que Australia podría colocar fácilmente en su mercado. Incluso ellos señalan que esta entrada se ha traducido en indices económicos que, en lugar de medir la realidad, miden los niveles económicos. El producto bruto del sector monetario se acrecentó en un $33 \%$ en 1970 pero ello no representa un crecimiento económico en el sentido amplio del téx-

${ }^{23}$ Declaración de prensa del Primer Ministro, 15 de diciembre de 1972.

${ }^{1 *} \mathrm{E}$. K. Fisk y Maree Tait, "Los problemas de la ayuda. ¿Un caso para dar menos?", Nueva Guinea, vol. 7, no 2, junio-julio de 1972. 
mino, porque no se está midiendo el crecimiento a largo plazo en la productividad de la economía, sino una ola "temporaria" de actividad. Tal fluctuación crea problemas en una economía pequeña y no necesita "maximizar el bienestar ni las entradas de la población nativa."

Existen otras diferencias básicas con lo que ciertos analistas mediocres llaman "paises subdesarrollados". Niugini es, como dice Fisk, un país agrícola en estado de "afluencia primitiva".

"La estructura económica que examinaremos es bastante usual en muchos aspectos. Tenemos un vasto sector de subsistencia estancada pero sorprendente sobre el cual ha sido transferido un sector monetario de rápida expansión pero relativamente pequeño y dentro del cual el crecimiento económico tiene lugar en un porcentaje alentador.

"La principal imagen que se desprende es la de un pais de bajos ingresos en el cual virtualmente toda la población tiene todo el alimento que desee, buenas resiclencias, de acuerdo a sus cánones tradicionales y gran cantidad de tiempo libre para sus fiestas, ceremonias y otros pasatiempos. Es una economía potencialmente viable y autosuficiente en el nivel de la afluencia primitiva, pero que depende casi totalmente de la ayuda exterior y de la importación de tecnología extranjera y del capital, para cualquier avance superior a este nivel primitivo."' 15

EL IMIPACTO DE LA INDUSTRIA PESADA

BOUGAINVILLE.

Existió una razón importante para la revisión del Plan de Desarrollo de 1968 dentro de los tres primeros años de su publicación: el impacto que tuvo para el país su primera empresa importante, la extracción de un gxan depósito cuprífero de baja ley en la Isla de Bougainville. Con un costo cercano a los 400 millones de dólares australianos, la operación minera es una de las más importantes del mundo. Su impacto en la economf́a de Niugini puede medirse por el hecho de que en este año triplicará por sí misma el total de lo exportado para el año 1968-69.10

${ }^{25} \mathrm{E}$. K. Fisk, "La estructura económica" en Nueva Guinea en el umbral, ANU Press, Canberra, 1966, ps. 23-24. Para un pronóstico postexior y más detallado, ver E. K. Fisk, "Desarrollo en la Melanesia rural" en Cambio y desarrollo en la Melanesia rural; quinto seminario, W'aigani, ed. Narion W. Ward, ANU Press, Canberra, 1972, p. 23.

${ }^{10 V}$ 'éase M. L. Treadgold, "Aspectos económicos". E1 Proyecto cuprífero de 
La minería, primordialmente aurífera, jugó un importante papel en la economía de Papua Nueva Guinea antes de la Ségunda Guerra Mundial, pero se agotaron las reservas conocidas y los costos crecientes hacen que el clesarrollo sea antieconómico. El oro constituía el $75 \%$ de las ganancias de exportación en 1939-40, pero pará el periodo $1965-66$ bajó al $2 \%$.

Este dramático y novedoso desarrollo tiene como escenario una isla a unos cientos de millas al este de la capital nacional. Bougainville tiene 130 millas cle largo, con un ancho que varía entre 20 y 40 millas; es una isla montañosa, con frecuente actividad volcánica y sísmica y con lluvias tropicales que exceden los 5.000 (200") milímetros anuales en algunas regiones. Su población indígena de 90.000 habitantes se distingue físicamente por sus pieles de tinte negroazulado de la otra parte cle la población de Papua Nueva Guinea, a quienes ellos se refieren como "pieles rojas". Son miembros de diferentes grupos tribales, con 17 idiomas distintos, entre los cuales: el contacto cultural ha ido imponiendo tanto el Pidgin como el. inglés.

La población de Bougainville ha tenido 70 años de creciente contacto con europeos; en un comienzo, los trabajadores reclutados en Queensland $y$ en las plantaciones de las islas del Pacífico, luego administradores alemanes, comerciantes, colonos y misioneros católicos maristas, desde 1880 hasta 1914, para finalizar con los australianos.

La isla fue escenario de las más trágicas batallas durante la II Guerra Nundial. En determinado momento los japoneses tuvieron más de 60.000 hombres en la isla; a raíz de un desembarco de fuerzas anfibias en Torokina, los americanos ubicaron a $33.000 \mathrm{hom}-$ bres en un pequeña playa e infligieron 5.000 bajas a los japoneses en una sola batalla periférica. La acometida australiana hacia el sur, en dirección a la principal base japonesa, en 1944, trajo como consecuencia 8.500 muertos en batalla, unidos a 9.800 japoneses que murieron por enfermedacles. La pequeña población nativa fué destrozada no solamente por la guerra sino por la reacción precedente de.los europeos.

"Exceptuando a los misioneros católicos, muchísimos europeos, dirigidos por el Oficial del Distrito en Kieta, se fueron de la isla. Es indudable que los aldeanos sintieron que habian sido abandonados por quienes alardeaban previamente de su superioridad dorada"'17.

Bougainville", p. 98 ff. en Enciclopedia de Papua Nueva Guinea. También en vol. 47, 1971: Informe económico de Australia y Nueva Zelandia.

${ }^{27} \mathrm{~J}$. Momis y E. Ogan, "Visión de Bougainville", en Cambio y desarrollo en la Melanesia rural, ed. Marion TW. Ward, ANU Press, Canberra, 1972, p. 107. 
Ni se le dio al pueblo una satisfacción a sus necesidades y expectativas, promovidas por discursos oficiales que les anunciaron una era dorada cle post-guerra, ni existió un sistema educacional estatal, sino escuelas misioneras en la isla, hasta 1961. Tampoco se dieron programas adecuados de extensión rural, a pesar de las riquezas que contiene la isla, hasta 1958." $1 \mathrm{~s}$

Un grupo de habitantes de Bougainville pidió a los miembros de una misión en visita de las Naciones Unidas, en 1962, que forzaran el abandono del poder administrativo de la isla, en manos de Australia, y lo cecliesen a los Estados Uniclos, porque denunciaron que habían sido tratados "como perros". Este resentimiento contra la lejana capital de Port Moresby no es privativo de Bougainville, ni menos aún representa un sentimiento anti-blanco: era una expresión muy gráfica del sentimiento generalizado en Niugini de que sería mucho mejor su vida, si los fondos se utilizasen en desarrollo. En todo caso, esta actitud ha sido un factor importante y de profundas implicancias en la vida política de Niugini.

Persiste el dilema sobre la necesidad cle un separatismo; hay un núcleo de habitantes que cada cierto tiempo piden la secesión, para pasar del federalismo al "gobierno de Bougainville" (lo que se traduce en una autonomía creciente de la estructura administrativa central). Ellos quisieran establecer una unión con las vecinas islas del protectorado Británico, las islas de Salomón, que, por su parte, estaban buscando formas de gobierno más representativas, decididas mediante plebiscito. La gran mayoría de tales medidas han sido propuestas por el gxupo de fanáticos del progreso por separado, de las distintas regiones de Papua Nueva Guinea. Tendremos que seguir esperando respuesta a lo que planteó Griffin, sobre la motivación exclusivamente sentimental de esa búsqueda de secesión. ${ }^{19}$

Justamente en esta isla la CRA Exploration Pty. Ltd. comenzó la explotación sistemática de los depósitos de "Porphyry Copper", en 1964. Se hizo un importante hallazgo, revisado en los cinco años siguientes; la construcción proyectada para la extracción, se empezó en 1969, terminánclose en 1972.

El depósito, con un porcentaje de $0.48 \%$ de mineral de cobre por tonelada larga, es muy bajo si lo comparamos con los niveles mundiales; en consecuencia se tuvo que utilizar métodos de extracción que requieren una gran inversión de capital.

Esta no es la primera vez que una compañía minera, en Australasia, ha debido proveer una infraestructura muy grande como parte

${ }^{28}$ Op. cit., p. 108.

"James Griffin, "Bougainville - Secesión o sentimiento de justicia", Boletin de Negocios, vol. 48, n: 9, febrero de 1972, Departamento de Educación Adulta, Universidad de Sydney. 
integral del desarrollo. En Bougainville tuvieron además el grave problema de la actividad sísmica, la gran pluviosidad, lo escarpado del territorio y la carencia absoluta de facilidades. Como resultado, se ha derivado la edificación de dos ciudades, una en las montañas del sitio de explotación, la otra en la costa, unidas por una gran autopista de 17 millas; aparte, se construyó un gran puerto, una enorme central hidroeléctrica y toda una estructura que facilita la trituración y el concentrado, con su correspondiente canalización. ${ }^{20}$

El costo total, aproximadamente 400 millones de dólares australianos, empequeñeció cualquier otro proyecto previo, en Niugini, de los cuales 136 millones; es decir el $36 \%$, lo representan las inversiones para infiaestructura. La fuerte formación de capital es posiblemente mayor a toda la inversión privada realizada en el sector monetario de la economía, en los nueve años que van desde 1960 a 1969.21

Desde el comienzo estaba claro que fueron los aspectos políticos y sociales del proyecto, por sobre los problemas técnicos, los cruciales. La compañía estaba consciente de los problemas que han tenido que enfrentar quienes se dediquen a la minería en Sudamérica, Africa y en cualquier lugar donde el avance tecnológico no se armoniza con los requerimientos sociales.

En 1966 el gobierno australiano anhelaba poder realizar un desarrollo mayor, que podría (de hecho lo fue) producir la evidencia de una actividad creciente, para demostrar ante las Naciones Unidas el buen cumplimiento de las recomendaciones del informe IBRD. La Administración de Nueva Guinea también se mostraba impaciente. La compañía explotadora, que había gastado más de 5 millones de dólares australianos en exploraciones y comprobaciones del depósito, sin otro respaldo contractual que una sola exploración permitida, necesitaba igualmente una base firme sobre la cual poder concretar el ansiado desarrollo.

El resultado fue que hubo meses de duras negociaciones, entre la Administración de Papua Nueva Guinea, los Departamentos Australianos de Territorios y de Tesorería y Finanzas, con la compañía. Se llegó a un acuerdo muy específico, tras el cual se redactó un proyecto de ley que pasó a la Asamblea de la nación en 1967.22

Como consecuencia, en el año 1972, vino un enorme desarrollo de la minería en una sección de la isla que antes estaba abandonada. El

${ }^{20}$ Las descripciones técnicas de la operación minera y la construcción han sido entregadas en varios periódicos comerciales y en Procedimientos, Instituto Australasiano de Mineria y Metalurgia, junio, de 1971.

${ }^{21}$ Treadgold, op. cit., p. 99.

${ }^{22}$ Ordenanza cuprifera de Bougainville, n9 79, 1967, Casa de la Asamblea, Port Moresby. 
James Byth / Niugini: una nación cuprifera en el Pacífico so ucercn a su independencir

potencial lísico y económico para una nación reciente, fue inmenso. $\mathrm{El}$ impacto social del cambio, que es inevitable incluso en las sociedades más sofisticadas frente a un proyecto de tal envergadura, ha sido por cierto importantísimo. Indudablemente los eternos críticos enfatizan los resultados negativos, pero está clemostrado que por más que se entregue una compensación generosa al reducido grupo de habitantes de Bougainville afectados por tal medida, ellos seguirán añorando su pasado tranquilo y seguro.

El Convenio de explotación minera ha sido duramente criticado a causa de su complejidad ${ }^{23}$. Muchas de estas críticas señalan el error que significa tomar este pacto como algo completo y definido, existiendo otros métodos alternativos de desarrollo. En concreto, este acuerdo dispone el más amplio porcentaje de beneficios para Niugini, en los primeros veinte años del proyecto y luego una proporción creciente de ganancias. Cualquier observador imparcial experimentado podría certificar que el convenio es muy favorable para la nación, en cuanto a la distribución de bienestax, si lo comparamos con los diversos convenios que se han establecido últimamente entre países en desarrollo y empresas de explotación minera.

Los lineamientos principales son:

I. El gobierno participa con el $20 \%$ de la compañia operante, la Bougainville Copper Pty. Limited; para ello se solicitó un préstamo en Australia. El gobierno nombra a dos directores de la Junta, y de este modo tiene acceso a los asuntos financieros y a las decisiones de la gerencia.

Por su parte, la compañía matriz emitió acciones para venderlas a la población de Niugini. Aproximadamente 9.000 compradores, individualmente o por grupos de tribus, casi todos lugareños, adquirieron cerca de 1.100 .000 acciones de la BMIL. En Australia no se efectuó ninguna emisión pública, pero 40.000 accionistas de las compañias patrocinantes Conzinc Rio Tinto de Australia Ltd. y NBHC Holdings compraron títulos de la BML.

II. Niugini recibe derechos sobre todas las exportaciones en un porcentaje del 1,25 del valor F.O.B. De esta entrada se entrega el $5 \%$ a los propietarios del terreno donde se realiza la operación; esta conquista fue el resultado de una batalla solitaria en que se em-

${ }^{23}$ Las críticas sobre el impacto social del proyecto, a menudo censurando a la administración de la época tanto como a la compañía, se encuentran en Momis y Ogan, op. cit.; Griffin, op. cit.; John Ryan, La tierra caliente; Enfoque de Nueva Guinea, Macmillan Australian Paperback, 1971. Una visión marxista ha sido preparada por Mitch Thompson, "Creciniento y Sud-desarrollo - El cobre de Bougainville y el nuevo imperialismo", Niugini Reader, 1972, Unión de Estudiantes Australianos, Melbourne. El capítulo pertinente, de Richard West, River of Tears, Earth Island, Londres, 1972, es igualmente polémico. 
barcó, en el año 1967, el miembro representante cle Bougainville ante el Parlamento, Mr. Paul Lapun, hoy ministro de Minería de la Coalición Nacional. (I a ley minera australiana, aplicable también en Niugini, básicamente el principio británico de que los depósitos minerales son propiedad del Estado antes que del propietario de la tierra.)

III. Después de tres años exentos de impuestos (lo cual fue necesario para pagar los préstamos en Eurodólares y en US dólares, que cubrieron más de 250 millones de dólares australianos invertidos en la etapa previa del proyecto) y con las reducciones normales, la. compañia paga hoy sus impuestos, que aumentan considerablemente a medida que avanza la explotación. Una compañía normal pagaba el $22,5 \%$ de sus entradas en impuestos, en la época del acuerdo; pero esta proporción subió, en cuatro años, hasta el $50 \%$ y luego gradualmente, hasta el $66 \%$.

Agregánse a estos los impuestos obtenidos por el gobierno, de los contratistas y de los 10.000 empleados en las empresas constructoras, que se aplicarán también a la fuerza de trabajo permanente y en los nuevos centros urbanos de Bougainville. Esta fuerza de trabajo es muy pequeña, si la comparamos a las de Chile o Zambia; ello se debe a la intensa operación de capital requerida por el bajo grado del mineral.

IV. El gobierno deseaba introducir un programa acelerado de educación y adiestramiento, lo cual tuvo gran aceptación en la compañía, influida por un estudio sobre conflictos laborales realizado en otros países en desarrollo. El centro de perfeccionamiento de la empresa, de un costo superior a los 1.6 millones de dólares australianos por año y con un costo de tuncionamiento de 1 millón de dólares australianos anuales, indican que estamos frente a un importante instituto técnico. Además de los niveles de aprendiz y comercial, la compañía patrocina estudiantes en las carreras tecnológicas y universitarias que se dictan en Niugini. Toda esta preparación no exige retribución y está dirigida a la creación de una buena cantidad de personal adiestrado que enriquezca el "Pool" nacional de cerebros.

V. Esta preparación ha llevado al reemplazo de los blancos por trabajadores de Niugini, en tal proporción que viene a desmentir los pesimistas pronósticos de los empleadores que tienen larga experiencia de trabajo en el país. En 1972 la compañía logró que el $72 \%$ de sus empleados fueran de Niugini, habiéndose fijado como objetivo para 1980, llegar al $90 \%$. Sería necesario poner en relieve, a quienes ya se están preguntando cuando serán los propios ciudadanos de Niugini quienes asuman el control superior de las empresas, que fue solamente en 1970 , con la entrega de titulos a los primeros 
graduados de la Universidad de Papua Nueva Guinea, que esta nación tan reciente tuvo sus primeros universitarios formados en ella. Estos son un reducido grupo de seis graduados en Artes y cuatro en Ciencias.

En cuanto a las universidades ultramarinas, han egresado de ellas unos cuantos graduados en actividades terciarias, principalmente los practicantes médicos preparados en Suva (Fiji).

Se encuentra visiblemente dentro de los intereses de la compañía tener el mayor equipo posible de gente del país. Realizarlo rápidamente puede crear una intensa competencia entre empleadores, de los cuales el gobierno resulta uno de los más interesados, ya que de tal modo se puede concentrar lä experiencia que, hasta hoy, es reducida. Ciertamente es dificil proporcionar, en el reducido término de los primeros años de operación de tal empresa, una experiencia administrativa que logre concentrar veinte años en cinco. Pero se está haciendo un esfuerzo con alentadores resultados. Se trata de evitar promover al personal por hacer favores o por deudas; como éste, hay muchos aspectos del acuerdo que tratan de cubrir, anticipándose a posibles dificultades futuras, las condiciones óptimas para no concitar las críticas muy justificadas que otras compañias suscitan, por sus abusos, en diversos países en desarrollo.

El própósito del convenio fue maximizar el flujo continuo de beneficios para el gobierno de Niugini, permitiendo así que compartiera, por medio de su participación igualitaria y una imposición creciente, las ganancias adicionales que pueden esperarse, ante las alzas en los precios mundiales del cobre.

También proveía, por medio de un descuento impositivo, del aliciente necesario para atraer préstamos bancarios hacia un país en desarrollo, hasta entonces un desconocido en los mercados financieros mundiales.

Finalmente, el fardo dependia de la mayor producción, lo cual hizo posibles negociaciones para contratos de venta por 15 años. El porcentaje anual de producción previsto era superior a las 160.000 toneladas cortas de cobre, más 500.000 onzas de oro contenidas en concentrados.

Considerando que las críticas a la minería internacional se basan, en gran parte, en ataques a la integración vertical y a la pérdida de valor agregado, que fue en el pasado experiencia común en los países sudamericanos, merece destacarse que los contratos suscritos por la compañia no benefician a sus subsidiarias o relaciones, sino que mantienen a prudente distancia a las fundiciones del Japón, Alemania Oriental y España. Las ventas se hacen en base a los precios del Mercado de Londres, con un mínimo garantizado de US\$ 30 centavos por libra, proveyendo asi la seguridad necesaria 
para los préstamos bancarios. No existe un precio de transferencia; tanto el impuesto habitual como la maquinaria de regulación de exportaciones y la presencia de directores nombrados por el gobierno, en la administración de la compañía, dan lugar a un clima de apertura y diálogo que son un anatema de las prácticas usuales en la empresa libre.

Los representantes del gobierno son aceptaclos libremente y con agrado por la compañía, cuyo propósito es ser un buen ciudadano, incorporado al interés nacional. Ciertamente esta es una frase que los australianos toman en serio, aunque provoque ironias en muchos lugares del mundo.

A fines de 1972, el Paxlamento aprobó directivas para futuros proyectos mineros, introclucidas por el Padre Momis, miembro regional por Bougainville, las cuales han sido perfeccionadas por las enmiendas introducidas por el más importante representante de Bougainville, el Ministro de Minería, Mr. Paul Lapun. Estas orientaciones no tienen carácter de leyes, aunque ciertamente tendrán influencia politica. Reflejan un espíritu nacionalista muy comprensible, habiendo tomado la compañia muchas de ellas para sus planes venideros. Junto con otros actos y declaraciones de la Coalición Nacional, son una manifestación del sentimiento natural de los ciudadanos de Niugini ante el cual la compañia debe mantenerse abierta.

Desgraciadamente, ciertas declaraciones propaladas con gran colorido por la prensa, respaldando el viejo axioma de que las malas noticias son buenas noticias, han hecho crecer la incertidumbre entre los inversionistas potenciales de ultramar. Posiblemente en los meses previos a la toma del poder por parte del gobiemo independiente, a fines de 1973, se hará notar una reducción de esta tensión, cuando se promulgue detalladamente la legislación sobre inversiones, redactada por la Coalición Nacional.

EL FUTURO DE NIUGINI

Niugini enfrenta su independencia con un poder enorme que nunca tuvieron muchas naciones pequeñas, para su desarrollo, en el mismo periodo de evolución. Tal es la entrada por dividendos, derechos de explotación, impuestos y obligaciones de la mina de Bougainville.

Treadgold señaló que se llegó a un avalúo oficial de $248.9 \mathrm{mi}-$ llones de dólares australianos netos, de entrada para el gobierno, como suplemento a las cobranzas para gastos fiscales, desde el período 
1969-70 hasta 1981-2.24 Tan importante contribución al erario nacional, que de otra manera dependería fuertemente de la ayuda australiana, puede lograr la superación del inevitable efecto de enclave de un capitalismo intensivo, en una comunidad rural.

Treadgold señaló que el efecto de demostración de un desarrollo mayor, alienta otros proyectos de envergadura, para exploración y desarrollo de la minería, e igualmente las diversas inversiones en los variados campos de la actividad humana, como industria maderera y la pescadería, áreas muy promisorias. Para que este desarrollo prosiga, tal como el del gran depósito de cobre que explota la Kennecott en Ok Tedi, en la región montañosa cerca de la frontera de Irian, depende del gobierno que fortalezca el clima positivo para la inversión, junto a orientaciones claras sobre sus planès de desarrollo.

Podemos ver a un país que se diferencia progresivamente de su pasado colonial; más influido por Populorum Progressio que por Lenin o Mao; manteniendo su tendencia natural a la comunidad por sobre la iniciativa individual y la responsabilidad, adquiriendo felizmente, una gran unidad nacional.

Seguramente surgirá un fuerte nacionalismo que va adoptando en su entusiasmo inicial, actitudes básicas de todos los miembros en desarrollo, ante las Naciones Unidas. Por ejemplo, puede esperarse que a las compañías mineras se les plantee la Resolución $2158^{\circ}$ (XXI), sobre el derecho inalienable de ejercitar la soberanía permanente sobre los recursos naturales y decidir su forma de explotación y comercialización.

También esperamos que lean el preámbulo: "...el capital extranjero, tanto público como privado, tiene una importante misión que cumplir, cuando lo requieren países en desarrollo... tomando precauciones para que exista una supervisión gubernamental sobre las actividades del capital foráneo, para asegurar su utilización en los intereses del desarrollo nacional". En Niugini se ha establecido claramente el control del estado para las nuevas y grandes inversiones extranjeras.

En todas las economias del mundo se acostumbraba a moverse en dirección del procesamiento amplio de productos de exportación, a pesar dé las dificultades para el control de calidad y la comercialización de muchos de ellos. Frecuentemente se expresa que Niugini debería hacer otro tanto, habiendo proyectado ya el gobierno los pasos necesarios para comenzar la exportación masiva de diversos productos primarios.

${ }^{24}$ M. L. Treadgold, Enciclopedia de Papua Nueva Guinea, p. 100. La tasación se basó en un precio del cobre de US\$ 55 centavos por libra. La definición de gastos públicos relacionada con el proyecto, posiblemente sea motivo de discusión. 
La compañía tiene obligación de consultar con el gobierno para el procesamiento del cobre, cuando sea necesario. Como lo han aprendido varios países sudamericanos y africanos, el procesamiento avanzado resulta caro, $y$, en una situación de mercado inseguro para el metal, tiene un riesgo financiero y técnico.

Será obvio, a su debido tiempo, para los críticos locales del desarrollo minero, como ya lo es, en los países que han debido conocer penosamente las realidades del potencial del mexcado, que el margen concebible de ganancias nacionales, con el cobre y demás minerales, no puede compararse con el del petróleo, sobre el cual se basa el éxito de OPEC; además, aunque la. Bolsa de Metales de Londres no sea una maravilla, es un instrumento de ventas bastante útil.

En Niugini, el gobierno y la compañia comparten, bajo común acuerdo, el retorno pequeño, por un precio bajo en la bolsa de Londres y las ganancias del alza de los precios. Los esfuerzos de CIPEC para eliminar lo efímero de los precios en el mercado del cobre, en un metal que está de hecho sujeto a una substitución competitiva, debe ser observado con interés y simpatías.

Históricamente, la negociación de los contratos a largo plazo con los compradores de concentrados, de preferencia a los de metal refinado han hecho posible el desarrollo de Bougainville; y ocupando con ello el vasto porcentaje de producción corriente, aparecería como materia lejana, toda decisión de acelerar el proceso para aumentarla.

Mr. Dominic Mulaisho, de Mindeco, el organismo para-estatal minero de Zambia, preguntó a una audiencia americana, el año pasado "¿Podríamos esperar que CIPEC llegue a ser en estos tiempos un instrumento para lograr una política de precios más racional?" No contestó su propia pregunta, porque quizás hasta hoy,. no consigue respuesta.

En Niugini también surgen interrogantes imposibles de responderse. Hank Nelson sintetizó un excelente, aunque algo pesimista estudio, de esta forma.

"A largo plazo, el pueblo de Niugini se hace confidente de uno. Tienen una cortesía, imaginación y fuerza práctica, buscando entregar sus propias soluciones. Aun sin instituciones de gobierno, saben. hacer su trabajo, aunque a veces tienen violentas guerras intestinas, para defenderse. Los australianos creian en la posibilidad de tener paz, estabilidad política y prosperidad económica, como una norma, pero de hecho no es habitual encontrarlas; más bien sería difícil predecir, al menos para un futuro de corto plazo, que en Niugini se logrará la paz y la prosperidad."

Esta es una visión sombría. Como australiano optimista, puedo 
James Byth / Niugini: una nación cuprifora on al Pacifico se acerca a su independencia esperar el surgimiento de una nación vecina, que se desarrolla en el Pacífico Occidental, siguiendo orgullosamente su propio camino pero aprendiendo de los exrores, tanto como de la ayuda de los demás países. 